\title{
A Case of Amyloid- $\beta$-Related Angiitis: Histology, CSF-Findings and Treatment
}

\author{
Susanne Hertel ${ }^{\mathrm{a}, \mathrm{d}}$, Thomas Eckey ${ }^{\mathrm{b}}$, Markus Glatzel ${ }^{\mathrm{c}}$, Andreas Moser ${ }^{\mathrm{a}}$
}

\begin{abstract}
Amyloid- $\beta$-related angiitis (ABRA) is characterized by vasculitis and accumulation of amyloid- $\beta$ in vessel walls. In this case report we describe findings of cranial MRI, brain biopsy and cerebrospinal fluid in a patient with Amyloid- $\beta$-related angiitis. The cranial MRI displayed extensive multifocal white matter lesion and brain biopsy revealed amyloid angiopathy as well as granulomatous angiitis. In the cerebrospinal fluid our patient showed normal cell count, slightly elevated protein and intrathecal immunoglobulin synthesis which recovered during steroid therapy. We illustrate that detailed CSF analysis including tests for intrathecal immunoglobulin synthesis is very useful in monitoring the efficacy of therapy and disease activity in ABRA since cranial MRI and histology are not suitable for this purpose. In addition we propose that CSF findings may be very helpful in diagnosing ABRA and starting early immunosuppressive therapy.
\end{abstract}

Keywords: Amyloid- $\beta$-related angiitis; Amyloid angiopathy; Cerebrospinal fluid analysis; Cranial MRI; Brain biopsy; Steroid therapy; Intrathecal immunoglobulin synthesis

\section{Introduction}

Recently, patients with Amyloid- $\beta$-related angiitis (ABRA) were described $[1,2]$. Brain biopsy revealed vasculitis and pronounced accumulation of amyloid- $\beta$ in vessel walls. Patients often present with cognitive change, headache and focal neurological deficits similar to other cerebral vasculitis

Manuscript accepted for publication August 6, 2012

\footnotetext{
${ }^{a}$ Department of Neurology, University of Luebeck, Germany

${ }^{\mathrm{b}}$ Institute of Neuroradiology, University of Luebeck, Germany

${ }^{\mathrm{c} I n s t i t u t e}$ of Neuropathology, University Hospital Hamburg, Germany

${ }^{\mathrm{d}}$ Corresponding author: Susanne Hertel, Department of Neurology,

University of Luebeck, Ratzeburger Allee 160, D-23538 Luebeck,

Germany. Email: susanne.hertel@neuro.uni-luebeck.de
}

doi: http://dx.doi.org/10.4021/jnr123w and are often treated with immunosuppressive therapy.

Here we report for the first time detailed inflammatory cerebrospinal fluid (CSF) findings and recovery during steroid therapy illustrating the importance of detailed CSF analysis in diagnosing and therapy monitoring of cerebral vasculitis.

\section{Case Report}

A 69-year-old woman developed progressive confusion, aphasia, disturbance of memory and headache over a one week period. Her medical history was significant for wellcontrolled hypertension and diabetes type 2, which was treated with diet. She did not have a history of dementia. There were no rheumatic diseases in her family, the patient's mother suffered from Parkinson's disease.

General physical examination was normal with blood pressure of $140 / 70 \mathrm{mmHg}$ and axillary temperature of $36.5^{\circ} \mathrm{C}$. On neurological examination she was not oriented to time, had short-term memory deficits, amnesic aphasia with dyslexia, dysgraphia and dyscalculia. Additionally she had paresis with increased tendon reflexes of the right arm.

Blood leucocytes were $10.000 / \mu$, C-reactive protein was normal. Blood analysis revealed slightly but not significantly elevated levels of antinuclear antibodies (ANA; 1:160) and antineutrophil cytoplasmic antibodies (ANCA; $1: 80)$.

Lumbar puncture revealed disturbance of the bloodbrain barrier with an increase of $\mathrm{CSF} /$ serum albumin ratio to $13.1 \times 10^{-3}$ (normal $<7.4 \times 10^{-3}$ ). Additionally, intrathecal synthesis of immunoglobulin (Ig) IgG (1\%), IgA (25\%), and $\operatorname{IgM}(7 \%)$ could be detected (Fig. 1). Oligoclonal IgG bands were found identically in CSF and serum. Cell count, glucose and lactate were normal. Amyloid- $\beta$ 1-42 protein $(354 \mathrm{pg} / \mathrm{ml})$, tau protein $(277 \mathrm{pg} / \mathrm{ml})$, and phosphorylated tau protein $(39 \mathrm{pg} / \mathrm{ml})$ were measured by standard ELISA technique and not different to normal values. All serum and CSF tests for infection were unremarkable, including human immunodeficiency virus types 1 and 2, BK virus and JC virus. Also anti-neural antibodies were negative in serum. CADASIL (Cerebral Autosomal Dominant Arteriopathy with Sub- 


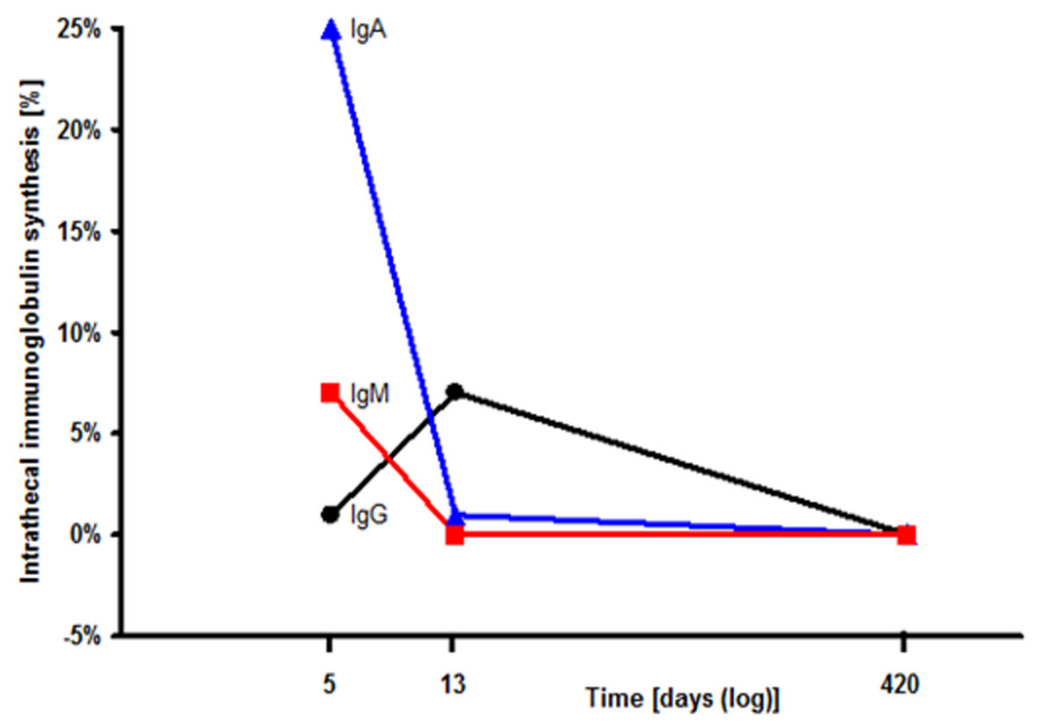

Figure 1. Intrathecal immunoglobulin synthesis in the cerebrospinal fluid (CSF): The CSF index for IgA (filled triangles), IgM (filled squares), IgG (filled circles) successively declined during steroid therapy.

cortical Infarcts and Leukoencephalopathy) was excluded by a gene test.

Cranial MRI showed besides multiple white matter lesions highly increased T2 signal in the left temporoparietooccipital area and the right temporal lobe with an extensive edema with a significant mass effect and midline shift. Susceptibility Weighted Imaging (SWI) MRI revealed multifocal black dots localized in the periphery of the left parietooccipital lobe (Fig. 2). No gadolinium enhancement or major haemorrhage could be detected.

A brain biopsy of the left occipital lobe revealed a cerebral amyloid angiopathy with perivascular cellular infiltration of the small vessels and perivascular microbleeds. Brain vessels presented with pronounced fibrosis of the walls and partial subtotal occlusion of the lumen. Immunohistochemistry demonstrated amyloid- $\beta$ in the walls of small and medium-sized parenchymal and leptomeningeal vessels (Fig. 3).

The patient was initially treated with $32 \mathrm{mg}$ dexamethasone per day and her clinical deficits ameliorated within days and she only had mild affective disturbance. Two weeks after the first lumbar puncture CSF/serum albumin ratio $\left(6.9 \times 10^{-}\right.$ ${ }^{3}$ ) as well as intrathecal immunoglobulin synthesis of $\operatorname{IgA}$ $(1 \%)$, and $\operatorname{IgM}(0 \%)$ declined while IgG synthesis slightly increased (Fig. 1). Cell count was normal and oligoclonal bands were negative in the CSF.

Once the clinical improvement had stabilized we tried to wean the steroids. Three weeks after cessation of steroids the patient showed acute worsening with short-term memory deficits, aphasia, apraxia and latent paresis of the right arm reoccurred, and she presented a new right hemianopsia. The cranial MRI revealed a progressive edema surrounding the left temporoparietooccipital lesions. Steroid therapy was re- started with dexamethasone (6 mg per day) and neurological symptoms improved within days, she presented only mild short-term memory deficits. The patient then received a long term dexamethasone therapy (5 mg per day) and cranial MRI after 6 month showed a clear regression of the edema and increased T2 signal in the affected brain areas.

After one year the neurological examination was unremarkable and lumbar puncture showed normal values for albumin, cell count and immunoglobulin in CSF (Fig. 1). We therefore decided to terminate steroid therapy. The patient did not take any immunosuppressive medication for 6 months by the time of writing this paper, and she is asymptomatic.

\section{Discussion}

In summary, our patient presented with subacute headache and neuropsychological deficits as disorientation, short-term memory deficits, amnesic aphasia with dyslexia, dysgraphia and dyscalculia as previous reported patients with Amyloid$\beta$-related angiitis (ABRA) [2-4]. The cranial MRI displayed extensive multifocal white matter lesion and oedema. Brain biopsy revealed amyloid angiopathy as well a granulomatous angiitis characteristic for ABRA $[3,5]$. To our knowledge this is the first report showing a detailed course of CSF changes over the time period of successful steroid therapy in a patient with ABRA. As described in previous case reports of ABRA, our patient showed normal cell count and slightly elevated protein indicating disturbance of the blood-brain barrier (BBB) $[2,3]$.

Patients with immunological diseases such as sarcoid- 

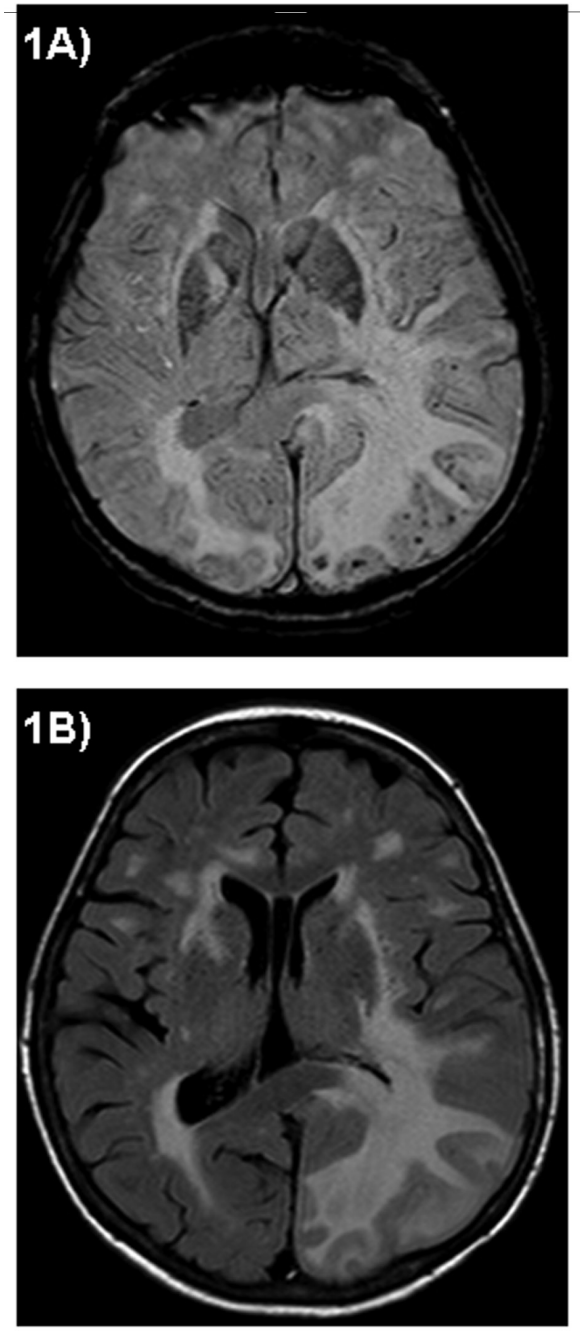

Figure 2. Axial Cranial MRI: Susceptibility Weighted Imaging (SWI) MRI displays multifocal black dots localized in the left parietooccipital lobe (A). Fluid Attenuated Inversion Recovery (FLAIR) MRI shows multiple white matter lesions particularly in the left parietooccipital area with an extensive edema and significant mass effect (B). osis, systemic lupus erythematosus or Behcet's disease involving the central nervous system (CNS) also show weakening of the BBB, intrathecal Ig synthesis or oligoclonal Ig bands in $20-50 \%$ [6]. Only little is, however, known on inflammatory CSF changes in patients with ABRA, but increased IgG was shown in the CSF in singular cases [1]. Most reports do not enclose information about examinations of the CSF (e. g. immunoglobulin).

In our patient, CSF analysis showed weakening of the $\mathrm{BBB}$ with elevated albumin and intrathecal synthesis of $\operatorname{IgA}$, IgM and IgG. The CSF findings reflecting the inflammatory process are in line with CSF results from patients with other inflammatory-immunological disorders affecting the CNS. These CSF changes normalized after initiation of steroid therapy. The second lumbar puncture revealed a decline of intrathecal synthesis of $\operatorname{IgG}$ and IgA. After having paused immunosuppressive therapy, patient's neurological deficits reoccurred within few days. The patient was treated again with steroids for one year and CSF completely normalized. Now the patient has stayed stable without immunosuppressive therapy for at least six months. White matter lesions in cranial MRI usually stay visible under immunosuppressive therapy and do not resolve in accordance to the clinical course.

We therefore conclude that detailed CSF analysis including tests for intrathecal Ig synthesis is very useful in monitoring the efficacy of therapy or disease activity in ABRA, since cranial MRI and histology are not sufficient for this purpose. In addition, we propose that CSF findings, beside clinical presentation, brain biopsy and cranial MRI may be very helpful in diagnosing ABRA and for starting immunosuppressive therapy early.

\section{Conflict of Interest}

The authors have no conflict of interest.
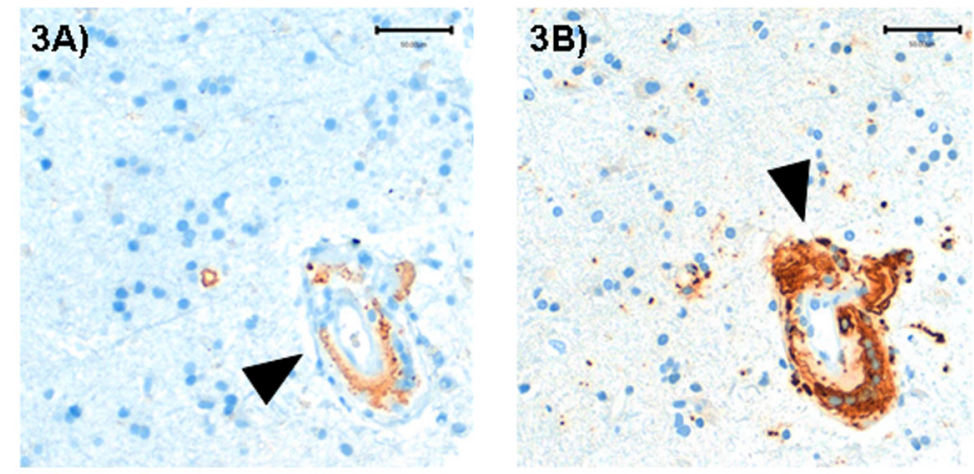

Figure 3. Brain biopsy (left occipital lobe): A medium sized vessel with granulomatous inflammatory infiltrate, deposition of amyloid- $\beta$ in vessel wall $(A$, immunohistochemical staining against amyloid- $\beta$, arrow) and multinucleated macrophages $(B$, immunohistochemical staining with the macrophage marker CD68, arrow). 


\section{Sources of Funding}

There was no funding other than that of the authors' institution.

\section{References}

1. Annweiler C, Paccalin M, Berrut G, Hommet C, Lavigne C, Saint-Andre JP, Beauchet O. Association of angiitis of central nervous system, cerebral amyloid angiopathy, and Alzheimer's disease: report of an autopsy case. Vasc Health Risk Manag. 2008;4(6):1471-1474.

2. Scolding NJ, Joseph F, Kirby PA, Mazanti I, Gray F, Mikol J, Ellison D, et al. Abeta-related angiitis: primary angiitis of the central nervous system associated with cerebral amyloid angiopathy. Brain. 2005;128(Pt 3):500-515.
3. Fountain NB, Lopes MB. Control of primary angiitis of the CNS associated with cerebral amyloid angiopathy by cyclophosphamide alone. Neurology. 1999;52(3):660662.

4. Gray F, Vinters HV, Le Noan H, Salama J, Delaporte P, Poirier J. Cerebral amyloid angiopathy and granulomatous angiitis: immunohistochemical study using antibodies to the Alzheimer A4 peptide. Hum Pathol. 1990;21(12):1290-1293.

5. Tschampa HJ, Niehusmann $P$, Marek M, Mueller CA, Kuchelmeister K, Urbach H. MRI in amyloid beta--related brain angiitis. Neurology. 2009;73(3):247.

6. McLean BN, Miller D, Thompson EJ. Oligoclonal banding of IgG in CSF, blood-brain barrier function, and MRI findings in patients with sarcoidosis, systemic lupus erythematosus, and Behcet's disease involving the nervous system. J Neurol Neurosurg Psychiatry. 1995;58(5):548554. 